\title{
Revista Española de
}

Nutrición Humana y Dietética

\section{Spanish Journal of Human Nutrition and Dietetics}

www.renhyd.org

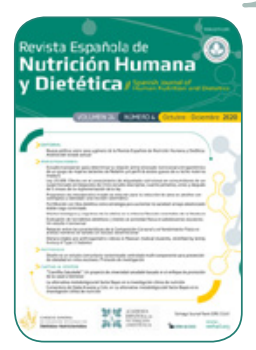

\section{ARTÍCULO DE INVESTIGACIÓN}

\section{Ley 20.606: Efectos en el conocimiento de etiquetado nutricional en consumidores de un supermercado en Valparaíso de Chile: estudio descriptivo, cuanticualitativo, antes y después de 5 meses de la implementación de la ley}

\author{
Mirta Crovetto ${ }^{a, *}$, Mytzi Acosta $^{a}$, Yuliza Rocco ${ }^{a}$ \\ ${ }^{\text {a }}$ Centro de Estudios Avanzados, Universidad de Playa Ancha, Valparaíso, Chile. \\ *mcrovetto@upla.cl \\ Editora Asignada: Carolina Aguirre-Polanco. Pontificia Universidad Católica de Chile. Santiago, Chile.
}

Recibido el 30 de diciembre de 2019; aceptado el 24 de julio de 2020; publicado el 1 de septiembre de 2020.

\section{PALABRAS CLAVE}

Obesidad;

Enfermedades no Transmisibles;

Etiquetado de Alimentos:

Legislación

Alimentaria;

Nutrientes;

Alimentos

Ley 20.606: Efectos en el conocimiento de etiquetado nutricional en consumidores de un supermercado en Valparaíso de Chile: estudio descriptivo, cuanticualitativo, antes y después de 5 meses de la implementación de la ley

\section{RESUMEN}

Introducción: El objetivo fue describir los cambios en la lectura, conocimientos e interpretación de los rótulos del etiquetado nutricional de los alimentos en los consumidores de un supermercado de Valparaíso, antes y después de la entrada en vigencia de la ley 20.606, entre junio y diciembre del año 2016.

Material y Métodos: Estudio descriptivo, no experimental, enfoque cuanti-cualitativo. Muestra por conveniencia de 200 consumidores del mismo supermercado a los cuales se les aplicó un cuestionario antes y después de la puesta en marcha de la ley con preguntas sobre conocimiento e interpretación de los rótulos "ALTO EN". Las respuestas se ordenaron en: i) contienen gran cantidad del nutriente/alto en el nutriente; ii) cantidad riesgosa para la salud; iii) más de lo necesario/sobre el nivel permitido; iv) tiene añadido el nutriente; v) no sé/no entiendo; vi) otros. Los cambios se evaluaron con diferencia de proporciones con un 95\% de intervalo de confianza.

Resultados: El incremento en la lectura del etiquetado nutricional (11\%) no fue significativo. Respecto al conocimiento e interpretación de los rótulos por los consumidores fue significativo para "ALTO EN CALORÍAS" en i y iii; para "ALTO EN SODIO" en i, ii y v; para "ALTO EN AZÚCAR" en ii y iii; y para "ALTO EN GRASAS SATURADAS" en i. Un 62\% dejó de elegir algún alimento por la cantidad de rótulos del envase. El 34\% señaló la necesidad de mayor educación sobre el nuevo etiquetado.

Conclusiones: Se generan cambios en la comprensión de los sellos "ALTOS EN" en el nuevo etiquetado, la lectura y la elección de los alimentos. Los consumidores indican que falta educación alimentaria y nutricional. A nivel de política de salud se requieren estudios cualitativos e indicadores para la evaluación de la comprensión e interpretación de los sellos a mediano y largo plazo y educación alimentaria y nutricional. 
Ley 20.606: Efectos en el conocimiento de etiquetado nutricional en consumidores de un supermercado en Valparaíso de Chile: estudio descriptivo, cuanticualitativo, antes y después de 5 meses de la implementación de la ley

\section{KEYWORDS}

Obesity;

Noncommunicable

Diseases;

Food Labeling;

Legislation, Food;

Nutrients;

Food.

Law 20,606: How it affects the knowledge about nutritional labeling of users of a supermarket in Valparaiso, Chile: A descriptive, quanti-qualitative study of before the implementation of the law and 5 months later

\section{ABSTRACT}

Introduction: The aim was to describe changes on the reading, knowledge and interpretation of the nutritional labeling of foods among consumers of a supermarket in Valparaiso, before and after the entry into force of Law 20,606, during June and December of 2016.

Material and Methods: Descriptive, non-experimental study, with a quantitative-qualitative approach. A convenience sample of 200 consumers who attended the same supermarket was surveyed using a questionnaire. The survey mode was an interview before and after the entry into force of the Law. The questionnaire included questions aimed to evaluate the knowledge and interpretation of the labels "HIGH IN". Responses were categorized as: i) It contains a great amount of the nutrient/is high in the nutrient; ii) The amount represents a risk for health; iii) It contains more than necessary/above the allowed level; iv) It has such nutrient added; v) I don't know/don't understand; vi) Other responses. Changes were assessed taking into account difference between proportions and confidence interval was set to $95 \%$.

Results: There was no significant increase in the reading of the nutritional labeling (11\%). Respecting the knowledge and interpretation of the labels, knowledge increased significantly regarding the label "HIGH IN CALORIES" in i and iii; regarding "HIGH IN SODIUM" in i, ii and v; regarding "HIGH IN SUGARS" in ii and iii; and regarding "HIGH IN SATURATED FATS" in i. Responses showed that $62 \%$ stated they have stopped consuming a certain food because of the number of stamps that the label contained and 34\% indicated the need for more education on the new labeling.

Conclusions: There were changes in the consumers' understanding of labels indicating "HIGH IN" of the new labeling system; reading of the labels and food choice were also modified. Consumers indicated that food and nutritional education is required. At the health policy level, qualitative studies and indicators are required to assess the understanding and interpretation of the seals in the medium and long term and food and nutrition education.

\section{CITA}

Crovetto M, Acosta M, Rocco Y. Ley 20.606: Efectos en el conocimiento de etiquetado nutricional en consumidores de un supermercado en Valparaíso de Chile: estudio descriptivo, cuanticualitativo, antes y después de 5 meses de la implementación de la ley. Rev Esp Nutr Hum Diet. 2020; 24(4): 311-23. doi: 10.14306/renhyd.24.4.979

\section{INTRODUCCIÓN}

Según el informe de la Organización para la Cooperación y el Desarrollo Económico (OCDE) 2017, Chile es uno de los países que se sitúa en los primeros lugares en el aumento de la malnutrición por exceso y el segundo país con la tasa más alta de obesidad entre los países miembros ${ }^{1}$.

La última Encuesta Nacional de Salud (ENS) 2017 mostró que un $74,2 \%$ de la población adulta presenta sobrepeso u obesidad, un $7,5 \%$ de incremento respecto a la ENS 2009-20102,3. En 2018, más de un 50\% de niños entre 6 a 7 años presentó sobrepeso u obesidad, un aumento del 0,6\% respecto al $2017^{4,5}$. Estos antecedentes revelan el problema de salud pública que tiene el país y el incremento de las enfermedades no trasmisibles (ENT) que en su mayoría se encuentran relacionadas con la dieta, factor de riesgo modificable y por tanto prevenible $e^{6,7,8}$.

La Organización Panamericana de la Salud (OPS) y la Organización Mundial de la Salud (OMS) señalan que el etiquetado frontal de alimentos es una estrategia efectiva, sencilla y fácil para fomentar los patrones de alimentación saludables, que en conjunto con otras acciones dirigidas a la regulación de ventas de productos en el entorno escolar y la regulación de la publicidad de alimentos, pueden disminuir el aumento de ventas de productos ultraprocesados ${ }^{9,10}$. 
Ley 20.606: Efectos en el conocimiento de etiquetado nutricional en consumidores de un supermercado en Valparaíso de Chile: estudio descriptivo, cuanticualitativo, antes y después de 5 meses de la implementación de la ley

Éstos contienen un alto contenido calórico y un bajo valor nutricional, se publicitan y comercializan de manera agresiva; son cultural, social, económica y ambientalmente destructivos y probablemente, son una de las principales causas alimentarias del aumento de peso y de las Enfermedades No Trasmisibles (ENT) ${ }^{11}$.

En relación a la composición de la dieta, estudios nacionales han señalado que un 55,2\% de la ingesta media de energía en sujetos mayores de 2 años o más, proviene de los alimentos procesados y ultraprocesados, representando estos últimos un $58,6 \%$ de la ingesta azúcares añadidos ${ }^{12}$. A nivel de gasto en alimentos, un estudio señala un aumento en el gasto absoluto y relativo en alimentos de los hogares del Gran Santiago ${ }^{13,14}$.

En los últimos años, el etiquetado frontal de alimentos Front of Package (FoP) se ha desarrollado como una de las estrategias que impulsan los organismos internacionales para contribuir a enfrentar la epidemia de la obesidad y la carga de enfermedad relacionada con la alimentación, con el fin de lograr que las personas puedan tomar decisiones informadas en la elección de los alimentos ${ }^{9,15}$.

En el año 2012, Chile aprobó la ley de etiquetado y publicidad de alimentos ${ }^{16,17}$. La ley 20.606 "Sobre Composición Nutricional de los Alimentos y su Publicidad" es una medida de salud pública cuyos objetivos se orientan a la protección de la salud, especialmente de niños, niñas y jóvenes ante el aumento de la malnutrición por exceso y ENT, derivadas principalmente de una mala alimentación ${ }^{18}$.

El sistema de etiquetado frontal de advertencia (EFE) fue el más fácil, claro y de mayor entendimiento, según estudios previos $^{19}$. Esta estrategia posiciona a Chile como pionero mundial al incluir este tipo de etiquetado, la restricción de la publicidad alimentaria a menores de 14 años, y la venta de productos con los rótulos en establecimientos de la educación de preescolares (parvularia) y básica. Estos rótulos de advertencia tienen la finalidad de permitir una selección informada de alimentos y la identificación en forma fácil de alimentos y productos alimenticios, cuyos aportes nutricionales superan los límites establecidos por el reglamento sanitario para calorías, azúcares, grasas saturadas y sodio ${ }^{18,20}$.

La ley 20.606 establece tres etapas para la implementación de los límites de los nutrientes a considerar en $100 \mathrm{~g}$ de productos sólidos y $100 \mathrm{~mL}$ en productos líquidos ${ }^{18}$. La primera etapa entró en vigencia el 26 de junio del 2016 con la inclusión de sellos octagonales, color negro, borde blanco y con un texto en su interior "ALTO EN", seguido de "CALORÍAS", "AZÚCAR", "GRASAS SATURADAS" y "SODIO". Posteriormente, el 27 de junio de los años 2018 y 2019 entró en vigencia la segunda y tercera etapa de la ley respectivamente, con reducciones progresivas en los nutrientes críticos que contempla la ley ${ }^{17,18}$.

El objetivo de este estudio fue describir los cambios en la lectura, conocimientos e interpretación de los rótulos del etiquetado de alimentos "ALTOS EN", en consumidores de un supermercado de Valparaíso, antes y después de 5 meses de la entrada en vigencia de la primera etapa de la ley 20.606 .

\section{$\longrightarrow$ \\ MATERIAL Y MÉTODOS}

Estudio de tipo descriptivo, de diseño no experimental, de enfoque cuanti-cualitativo. La población estudiada corresponde a consumidores de un supermercado de fácil acceso emplazado en el centro de Valparaíso, Chile. Muestra por conveniencia conformada por 200 consumidores de un supermercado (100 antes -cuarta semana de junio de 2016-y 100 después de 5 meses de la entrada en vigencia de la ley 20.606 -diciembre de 2016-). El tamaño de la muestra por conveniencia se determinó en base al día y los horarios establecidos por el supermercado que autorizó la aplicación del cuestionario, con un máximo de usuarios por vez (limitante del estudio). Criterios de inclusión: ser usuario del supermercado de Valparaíso en los días de realización de la encuesta, sujetos de ambos sexos y edad igual o superior a 18 años y que accedieron en forma voluntaria a participar y responder el cuestionario previa explicación de la finalidad del estudio, agradeciendo su participación. Se consideró como criterio de exclusión los cuestionarios que no se llevaron a término por parte de los usuarios.

Variables: i) dependiente - conocimiento e interpretación de los rótulos por los consumidores; ii) independiente - etiquetado frontal de advertencia "ALTO EN".

La unidad de análisis fue los cuestionarios respondidos en su totalidad por los entrevistados antes y después de la puesta en marcha de la primera etapa de la ley 20.606 de etiquetado aplicados en modo de entrevista semiestructurada.

Los cuestionarios fueron elaborados por los autores y sometidos a juicio de expertos. Se incluyeron antecedentes generales del encuestado y preguntas relacionadas del etiquetado de alimentos. El primer cuestionario consistió en 22 preguntas con respuestas dicotómicas y abiertas (a fin de no inferir en la respuesta del consumidor) sobre el conocimiento de alimentos altos en los nutrientes críticos del etiquetado de alimentos en vigencia a la a fecha; 
Ley 20.606: Efectos en el conocimiento de etiquetado nutricional en consumidores de un supermercado en Valparaíso de Chile: estudio descriptivo, cuanticualitativo, antes y después de 5 meses de la implementación de la ley

el segundo cuestionario, incluyó preguntas similares al primero con el cambio de la leyenda "altos en" a otra, sobre qué entiende al ver los rótulos "ALTO EN CALORÍAS", "ALTO EN AZÚCAR", "ALTO EN SODIO" y "ALTO EN GRASAS SATURADAS" en los alimentos.

La recolección de los datos se realizó en dos ocasiones, la cuarta semana de junio (entre los días 20 y 26 de 2016) antes de la puesta en marcha de la ley 20.606 y 5 meses después de la entrada en vigencia de ésta, durante la primera semana de diciembre (entre los días 5 y 11 de 2016).

Se ordenaron las respuestas y se agruparon considerando las frases más repetidas en cada una de las preguntas para obtener patrones de respuestas que se ordenaron en las siguientes categorías: i) contiene gran cantidad del nutriente/alto en el nutriente; ii) cantidad riesgosa para la salud; iii) más de lo necesario/sobre el nivel permitido; iv) tiene añadido el nutriente; v) no sé/no entiendo; vi) otros.
La tabulación, el procesamiento y el análisis de datos (cálculo de porcentajes) se realizó con el programa Microsoft Excel 2010. Para el análisis estadístico de resultados, se utilizó la prueba de diferencia de proporciones con un 95\% de Intervalo de Confianza (IC).

\section{RESULTADOS}

Antecedentes generales de la muestra: La distribución por género en la encuesta 1 y 2 varía en un $7 \%$ manteniéndose cerca al 50\%; se observa un $60 \%$ en el rango de edad de 18 a 35 años en promedio de ambas encuestas; el $47 \%$ de la muestra tiene hijos menores de 14 años en promedio de ambas encuestas; el $70 \%$ presenta formación universitaria (Tabla 1).

Tabla 1. Antecedentes generales de la muestra. Encuesta 1 y Encuesta 2. Junio y diciembre, año 2016.

\begin{tabular}{|c|c|c|}
\hline & $\begin{array}{c}\text { Encuesta 1. Junio } 2016 \\
n^{(1)} y_{\%}^{(2)}\end{array}$ & $\begin{array}{c}\text { Encuesta 2. Diciembre } 2016 \\
n^{(1)} \text { y \% }\end{array}$ \\
\hline \multicolumn{3}{|l|}{ Género } \\
\hline Femenino & 46 & 53 \\
\hline Masculio & 54 & 47 \\
\hline Total & 100 & 100 \\
\hline \multicolumn{3}{|l|}{ Edad } \\
\hline 18-35 años & 59 & 61 \\
\hline 36-64 años & 39 & 39 \\
\hline$>65$ años & 2 & 0 \\
\hline Total & 100 & 100 \\
\hline \multicolumn{3}{|c|}{ Consumidores con hijos menores de 14 años } \\
\hline Sí & 54 & 40 \\
\hline No & 46 & 60 \\
\hline Total & 100 & 100 \\
\hline \multicolumn{3}{|c|}{ Nivel educacional ${ }^{(3)}$} \\
\hline Básica ${ }^{(I)}$ & 1 & 5 \\
\hline Media(II) & 29 & 25 \\
\hline Superior ${ }^{(I I I)}$ & 70 & 70 \\
\hline Total & 100 & 100 \\
\hline
\end{tabular}

n: Total consumidores; $\%$ : porcentaje.

(1) $\mathrm{n}$ : Cantidad de consumidores de un total de 100 consumidores.

(2) \%: Se unifican valores, dado que el total de consumidores son 100 .

(3) Fuente: Ministerio de educación.

(I) Fuente: Básica (primaria): Desde los 6 años, incluye 8 grados.

(II) Fuente: Media (secundaria): Desde los 14 años, incluye 4 grados.

(III) Fuente: Superior: Universitaria, instituto profesional y centros de formación técnica. 
Lectura del etiquetado nutricional frontal: La lectura del etiquetado nutricional por parte de los encuestados, mostró valores de (59\% y 70\% para la encuesta 1 y la 2, respectivamente (Figura 1). Esta diferencia no es significativa (95\%IC: -0,0217 a 0,2417). Respecto a las razones de lectura, las principales corresponden a "informarse", (33\%) "alimentarse saludablemente" (5\%) y "me interesa" (4\%) que se incrementan en un $6 \%, 15 \%, 2 \%$, respectivamente, en la encuesta 2, encontrándose una diferencia significativa para "alimentarse saludablemente" (IC95\%: 0,0607 a 0,2392), mientras que "en ocasiones" y "otros" se reducen en un $5 \%$ y $2 \%$, respectivamente. Por el contrario, las razones de no lectura disminuyen en la encuesta 2 y corresponden a "no entiendo" (IC95\%: 0,0196 a 0,2003) y "no me interesa" en un $11 \%$ y $9 \%$, respectivamente, mientras que la falta de tiempo" se mantiene (8\%) y "poca costumbre" aumenta de un $5 \%$ a un $7 \%$ (Tabla 2 ).

Los principales alimentos de interés nutricional informados por los consumidores en la encuesta 1 fueron "todos", "cereales" y "comida chatarra". En la encuesta 2, aumenta en un $32 \%$ "todos", por el contrario, disminuyen un 6\% "cereales" y en 10\% "ninguno" y "comida chatarra" (Figura 2).

En la pregunta "¿Cree usted que es dañino para la salud consumir alimentos que tengan un mayor aporte de calorías, azúcares, sodio y grasas saturadas?" sobre el $90 \%$ de
Figura 1. Cambios en la lectura del etiquetado nutricional de los alimentos, antes y después de la implementación de la ley 20.606. Junio y diciembre, año 2016.

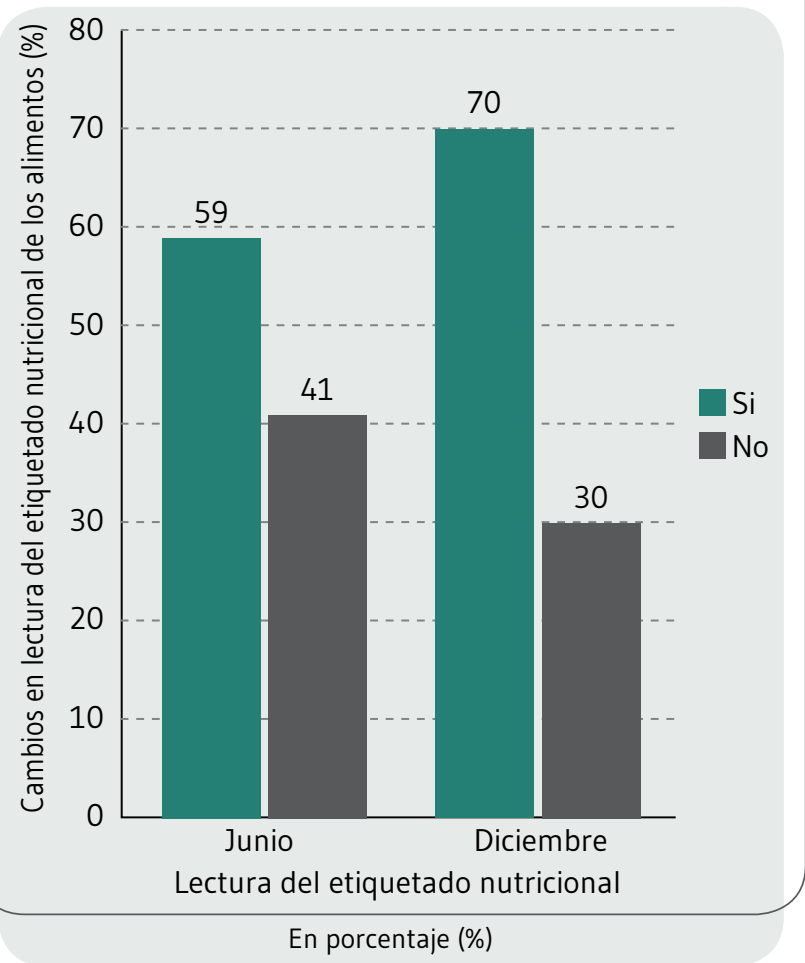

Tabla 2. Cambios en las razones de lectura y no lectura del etiquetado nutricional de los consumidores. Antes y después de la entrada en vigencia de la ley de alimentos 20.606. Junio y diciembre, año 2016.

\section{Razones}

\section{De lectura}

Informarse

Alimentarse saludablemente

Me interesa

En ocasiones

Por el cuidado de los niños

Otros

\section{De no lectura}

No entiendo

No me interesa

Falta de tiempo

Poca costumbre

Total
Encuesta 1. Junio 2016 $n^{(1)} y \%^{(2)}$

\section{Encuesta 2. Diciembre 2016 $n^{(1)} y \%^{(2)}$}

IC(3)

Encuesta 1 y 2

\begin{tabular}{l|c|c}
\hline 33 & 39 & $-0,0727$ a 0,1927 \\
\hline 5 & 20 & 0,0607 a 0,2392 \\
\hline 4 & 6 & $-0,0403$ a 0,0803 \\
\hline 6 & 1 & $-0,0004$ a 0,1004 \\
\hline 1 & 4 & $-0,0130$ a 0,0730 \\
\hline 3 & 0 & $-0,0034$ a 0,0634 \\
\hline 18 & & \\
\hline 17 & 7 & 0,0196 a 0,2003 \\
\hline 8 & 8 & $-0,0008$ a 0,1808 \\
\hline 5 & 8 & $-0,0751$ a 0,0751 \\
\hline 100 & 100 & $-0,0457$ a 0,0857 \\
\hline
\end{tabular}

n: Total consumidores; \%: porcentaje; IC: Intervalo de Confianza.

(1) $\mathrm{n}$ : Cantidad de consumidores de un total de 100 consumidores.

(2) \%: Se unifican valores, dado que el total de consumidores son 100 .

(3) IC: $95 \%$. 
Figura 2. Cambios en las respuestas de los consumidores respecto a los alimentos de interés nutricional. Antes y después de la implementación de la ley de alimentos 20.606. Junio y diciembre, año 2016.

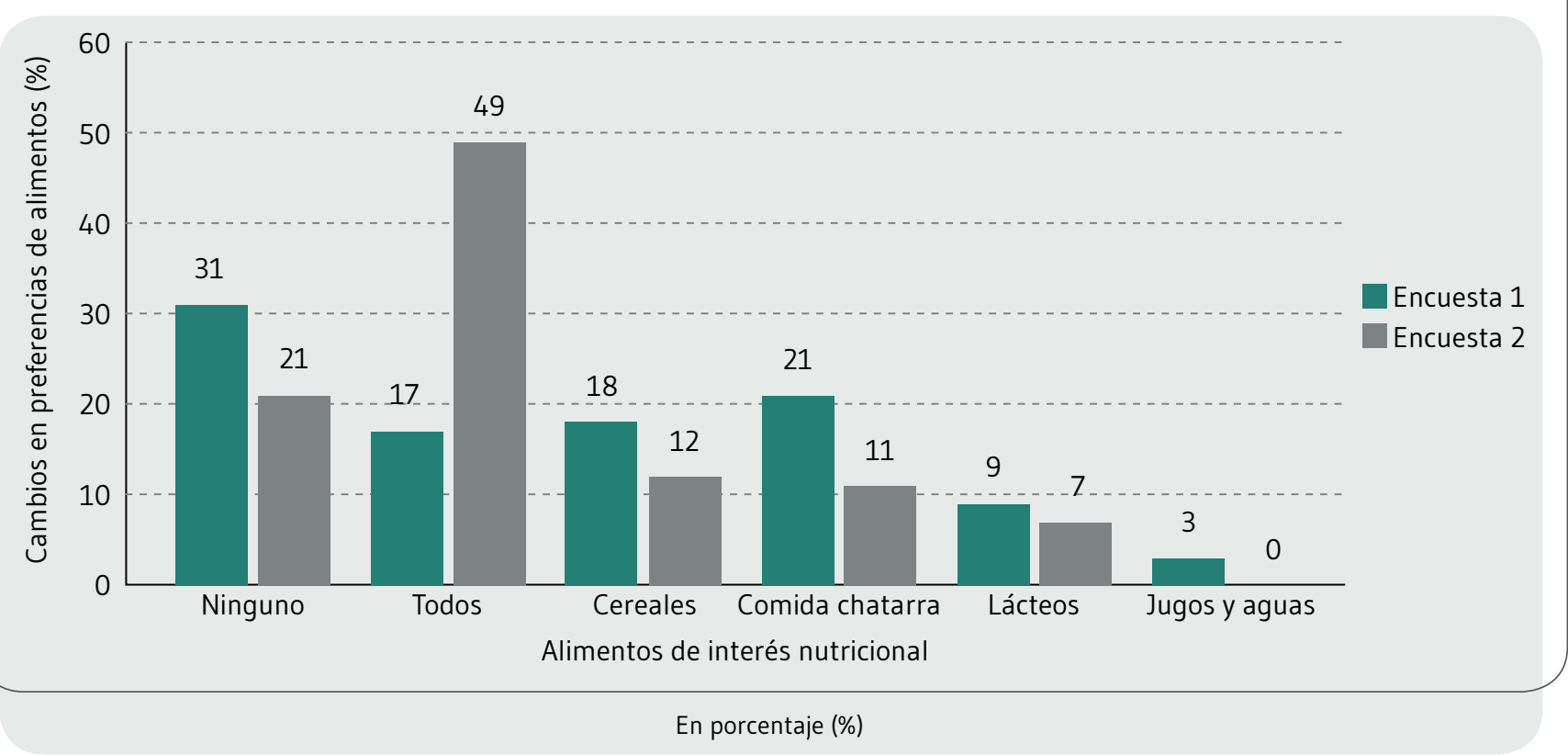

los consumidores afirman esta condición para los rótulos "ALTO EN" "AZÚCAR", "SODIO" y "GRASAS SATURADAS" en ambas encuestas, destacando que el rótulo "ALTO EN CALORÍAS" es al que menos asocian a este daño (70\% promedio entre ambas encuestas) (Figura 3).

Las principales razones de daño a la salud de los rótulos "ALTO EN", "CALORÍAS", "AZÚCAR", "SODIO" y "GRASAS SATURADAS" señaladas por los consumidores fueron: "producen sobrepeso y obesidad"; "producen ENT"; producen diabetes mellitus e insulino resistencia"; "producen HTA", que disminuyen en un $6 \%, 11 \%, 24 \%, 3 \%$ y $31 \%$, en la encuesta 2 , respectivamente. Por el contrario, aumenta en un 3\%, la razón "causa daño renal" para el rótulo "ALTO EN SODIO"; en un $4 \%$ y $8 \%$ para las razones "problemas cardiovasculares" y "aumentan el colesterol" para el rótuIo "ALTO EN" "GRASAS SATURADAS", respectivamente en la encuesta 2. Se encontraron diferencias significativas en el rótulo "ALTO EN CALORÍAS" para las razones "producen ENT"; en el rótulo "AZÚCAR" para las razones producen " diabetes mellitus e insulino resistencia" y "producen daño sólo en exceso"; en el rótulo "GRASAS SATURADAS" para la razón "producen sobrepeso y obesidad" (Tabla 3).

\section{Conocimiento en interpretación de los rótulos "ALTO EN":}

En la pregunta "¿Qué entiende respecto al rótulo "ALTOS EN" "CALORÍAS", "AZÚCAR", "SODIO", y "GRASAS SATURADAS" en los alimentos?". Las principales interpretaciones en los rótulos "ALTO EN" fueron en la categoría i (contiene gran cantidad del nutriente/alto en el nutriente) que aumenta en la encuesta 2 en un 26\% para "CALORÍAS", un $23 \%$ para "SODIO" y un 23\% para "GRASAS SATURADAS" y disminuye en un $13 \%$ " para "AZUCAR"; en la categoría ii (cantidad riesgosa para la salud), disminuye en un $12 \%$ para "AZÚCAR" en la encuesta 2; en la categoría iii (más de lo necesario/sobre el nivel permitido) aumenta en un $23 \%$ y en un 7\% para "AZÚCAR" y "SODIO", respectivamente, en la encuesta 2. En la categoría v (no sé/no entiendo) disminuye en la encuesta 2 para "CALORÍAS", "SODIO" y "GRASA SATURADAS" en un $8 \%$, un $5 \%$ y un $7 \%$, respectivamente. La categoría vi (otros), disminuye para "CALORÍAS" y "GRASAS SATURADAS" en un $4 \%$ y $12 \%$, en la encuesta 2 , respectivamente (Tabla 4). Se encontraron diferencias significativas en la categoría i; para los rótulos "ALTO EN" "CALORÍAS", "SODIO", "GRASA SATURADAS"; categoría ii para "AZÚCAR" $y$ "SODIO"; categoría iii para "CALORÍAS" y "AZÚCAR"; categoría v para "SODIO" (Tabla 4).

Efectos de la ley después de su implementación: Posterior a cinco meses de la puesta en marcha de la ley 20.606, un $62 \%$ de los consumidores manifiesta que dejó de consumir algún producto debido a la cantidad de rótulos que presenta (Figura 4).

Respecto a la pregunta "¿Qué cambios o mejoras realizarían al nuevo etiquetado nutricional?", un 34\%, 31\% y $22 \%$ 
Figura 3. Cambios en el conocimiento de los consumidores sobre el daño en la salud de los alimentos que contienen el rótulo "ALTO EN" "CALORÍAS", "AZÚCAR", "SODIO" y "GRASAS SATURADAS", antes y después de la implementación de la ley de alimentos 20.606. Junio y diciembre, año 2016.

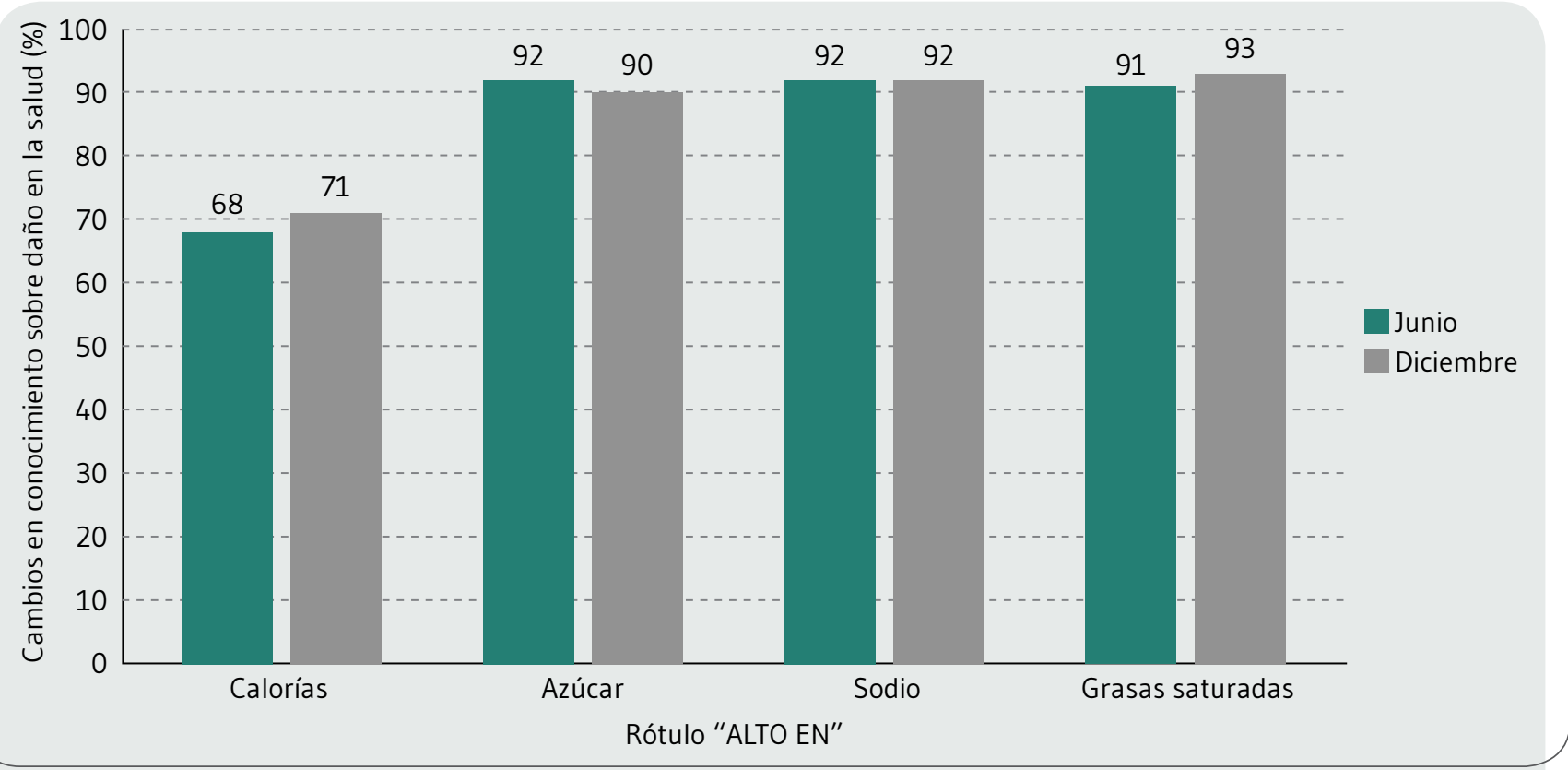

En porcentaje (\%)

indicaron "informar más a la población acerca del nuevo etiquetado", "realizar cambios en su imagen como tamaño, logos y formato" y "ningún cambio".

\section{DISCUSIÓN}

El etiquetado frontal de alimentos ha sido reconocido como una estrategia que contribuye a un mejor entendimiento de los consumidores sobre la calidad nutricional de los productos y que puede influir en la elección de compra en productos más saludables ${ }^{15,21}$. Chile ha sido pionero en la implementación de un etiquetado frontal de advertencia con las imágenes de octágonos negros que permiten identificar en forma muy simple los alimentos de alta densidad energética, azúcar, grasa saturada y sodio ${ }^{20}$. Un estudio previo para la decisión del uso de los octágonos en Chile mostró que este tipo de etiqueta era fácil de visualizar, comprender y contribuye a lograr cambios en la elección de compra ${ }^{19,22}$.

En Chile, dos meses antes de la entrada en vigencia de la primera etapa de la ley en 2016, una encuesta en el Gran
Santiago señaló que 7 de cada 10 personas indicó leer el etiquetado de alimentos, de los cuales un $50 \%$ observa que la lectura de éste influye en su decisión de compra ${ }^{23}$. Siete meses posteriores a su implementación, un estudio en adultos observa que un $43,8 \%$ y un $67,8 \%$ compara y escoge productos con menos cantidad de rótulos, un $91,6 \%$ y un $92,9 \%$ señala que influyen en la compra y entienden la información, respectivamente ${ }^{24}$. Estos resultados son similares a nuestro estudio en que cinco meses después de la puesta en marcha de la ley, un $70 \%$ declara leer el etiquetado nutricional, sin embargo, a pesar del incremento porcentual en la encuesta 2 no es proporcionalmente significativo (95\%IC: $0,0217$ a 0,2417$)$. Por otra parte, se observa una disminución de un $72 \%$ a un $66 \%$ entre la encuesta 1 y 2 , respecto a la comparación en la lectura del etiquetado nutricional, pudiendo interpretarse que los rótulos frontales están facilitando la identificación de alimentos menos saludables en forma más fácil. Un $62 \%$ de la muestra de este estudio manifestó escoger un producto debido a la cantidad de rótulos que presentan.

El Ministerio de Salud (MINSAL), posteriormente a la implementación de la primera etapa de la ley, en evaluaciones realizadas a distintos grupos de población, observó que un $92,4 \%$ está de acuerdo con el etiquetado obligatorio de 
Ley 20.606: Efectos en el conocimiento de etiquetado nutricional en consumidores de un supermercado en Valparaíso de Chile: estudio descriptivo, cuanticualitativo, antes y después de 5 meses de la implementación de la ley

Tabla 3. Cambios de los consumidores en las principales razones del daño a la salud en el consumo de productos "ALTO EN" "CALORÍAS", "AZÚCAR", "SODIO" y "GRASAS SATURADAS". Antes y después de la entrada en vigencia de la ley de alimentos 20.606. Junio y diciembre, año 2016.

\begin{tabular}{|c|c|c|c|c|c|}
\hline Rótulo “ALTO EN" & Razones & $\begin{array}{c}\text { Encuesta } 1 \\
\%\end{array}$ & $\begin{array}{c}\text { Encuesta } 2 \\
\%\end{array}$ & $\begin{array}{c}\text { Variación } \\
\%\end{array}$ & $I^{(1)}$ \\
\hline \multirow{2}{*}{ Calorías } & Producen Sobrepeso y obesidad & 37 & 31 & 6 & $-0,0710$ a 0,1910 \\
\hline & Producen $\mathrm{ENT}^{(2)}$ & 12 & 1 & 11 & 0,0433 a 0,1766 \\
\hline \multirow{4}{*}{ Azúcar } & Aumentan las grasas & 11 & 5 & 6 & $-0,0147$ a 0,1347 \\
\hline & Producen diabetes e insulino resistencia & 75 & 51 & 24 & 0,1103 a 0,3696 \\
\hline & Producen sobrepeso y obesidad & 10 & 14 & 4 & $-0,0499$ a 0,1299 \\
\hline & Afectan a la salud en exceso & 0 & 18 & -18 & 0,1046 a 0,2553 \\
\hline \multirow{3}{*}{ Sodio } & Producen $\mathrm{HTA}^{(3)}$ & 48 & 45 & 3 & $-0,1081$ a 0,1681 \\
\hline & Daño renal & 12 & 15 & -3 & $-0,0646$ a 0,1246 \\
\hline & Pone en riesgo la salud & 9 & 10 & -1 & $-0,0712$ a 0,0912 \\
\hline \multirow{3}{*}{ Grasas saturadas } & Problemas cardiovasculares & 16 & 20 & -4 & $-0,0663$ a 0,1463 \\
\hline & Producen sobrepeso y obesidad & 49 & 18 & 31 & 0,1864 a 0,4335 \\
\hline & Aumentan el colesterol & 11 & 19 & -8 & $-0,0183$ a 0,1783 \\
\hline \multicolumn{6}{|c|}{$\begin{array}{l}\text { \%: porcentaje; IC: Intervalo de Confianza. } \\
\text { (1) IC: } 95 \% \text {. } \\
\text { (2) Enfermedades No Transmisibles. } \\
\text { (3) Hipertensión Arterial. }\end{array}$} \\
\hline
\end{tabular}

sellos de advertencia; un 91,3\% sobre la prohibición de la venta en los colegios; un $74,5 \%$ en la prohibición de la publicidad de productos con rótulos 24,25 . Otro estudio en niños de 8 a 12 años de diferentes regiones del país, informó que el $53 \%$ de los niños de nivel socioeconómico (NSE) medio-alto y el $48 \%$ de NSE bajo, dejarían de comprar los alimentos con rótulos, mientras que un $87,3 \%$ y $78,5 \%$ de los niños de NSE medio-alto y bajo, respectivamente, señaló que les gustaba ser informados del contenido de los alimentos $(p<0,01)^{26}$. Otro estudio estimó que la proporción de productos con cero rótulos de advertencia entre la fase inicial en 2016 y la final en 2019 , disminuiría de un $38 \%$ a $17 \%$, respectivamente ${ }^{27}$. A su vez, el estudio realizado por tres universidades (Chile, Diego Portales y Carolina del Norte de Estados Unidos) indicó cambios en la percepción y actitudes de las madres sobre los sellos frontales de advertencia ${ }^{28}$.

Posterior a la implementación del etiquetado frontal de advertencia en Chile, a nivel mundial se han realizado estudios para evidenciar qué sistemas de etiquetado pueden ser los más efectivos para la elección de productos más saludables y con ello contribuir a la reducción de la obesidad. América Latina se presenta como la región que más ha legislado e implementado el etiquetado frontal obligatorio de alimentos; Ecuador y Bolivia optaron por el semáforo en los años 2014 y 2015, respectivamente; Perú y Uruguay con el etiquetado frontal de advertencia en el año 2018; Brasil inició con estudios que compararon distintos sistemas de etiquetado en los años 2016 y 2017, concluyendo que el sistema de advertencia puede lograr una mejor percepción sobre los productos más saludables y mejorar la intención de compra de la población ${ }^{29-33}$.

En Europa, Países Bajos y Estados Unidos han comparado distintos sistemas de etiquetados frontales para evaluar la eficacia en la percepción de los consumidores sobre los conocimientos e interpretación de éstos a la hora de reconocer los productos más saludables y el predominio en la decisión de compra para incorporar el etiquetado en los productos a pesar de que en estos países el etiquetado es voluntario ${ }^{34-37}$

Otros estudios, como el realizado en 12 países de distintas regiones del mundo (12.015 personas) en el año 2019 que comparó el efecto de cuatro tipos de etiquetas respecto al aumento del conocimiento sobre la calidad nutricional de productos, mostró que las etiquetas frontales interpretativas (Nutri-Score, semáforos múltiples, Health Star Rating (HSR) y el símbolo de advertencia, este último implementado en Chile) superaron significativamente al etiquetado 
Tabla 4. Cambios en el conocimiento de los descriptores "ALTO EN" "CALORÍAS", "AZÚCAR", "SODIO" y "GRASAS SATURADAS por categorías de respuesta. Antes y después de la entrada en vigencia de la ley de alimentos 20.606. Junio y diciembre, año 2016.

\begin{tabular}{|c|c|c|c|c|c|c|c|c|c|c|c|c|}
\hline \multirow[b]{2}{*}{ Categorías } & \multicolumn{4}{|c|}{$\begin{array}{l}\text { Encuesta } 1 \\
\text { Junio } 2016\end{array}$} & \multicolumn{4}{|c|}{$\begin{array}{c}\text { Encuesta } 2 \\
\text { Diciembre } 2016\end{array}$} & \multicolumn{4}{|c|}{$\begin{array}{c}I^{(3)} \\
\text { Encuestas } 1 \text { y } 2\end{array}$} \\
\hline & $\begin{array}{l}\text { Calorías } \\
N^{(1)} \text { y } \%^{(2)}\end{array}$ & $\begin{array}{c}\text { Azúcar } \\
N^{(1)} \text { y \%(2) }\end{array}$ & $\begin{array}{c}\text { Sodio } \\
N^{(1)} \text { y \%(2) }\end{array}$ & $\begin{array}{c}\text { Grasas } \\
\text { saturadas } \\
\mathbf{N}^{(1)} \mathrm{y}^{(2)}\end{array}$ & $\begin{array}{l}\text { Calorías } \\
\mathbf{N}^{(1)} \text { y \% }\end{array}$ & $\begin{array}{c}\text { Azúcar } \\
\mathrm{N}^{(1)} \text { y \%(2) }\end{array}$ & $\begin{array}{c}\text { Sodio } \\
N^{(1)} y \%^{(2)}\end{array}$ & $\begin{array}{c}\text { Grasas } \\
\text { saturadas } \\
\mathrm{N}^{(1)} \text { y } \%^{(2)}\end{array}$ & Calorías & Azúcar & Sodio & $\begin{array}{l}\text { Grasas } \\
\text { saturadas }\end{array}$ \\
\hline
\end{tabular}

Contiene gran

cantidad del nutriente/principa ingrediente/alto en \begin{tabular}{l|l:l:l}
22 & 57 & 41 & 15
\end{tabular}

48

44

64

38

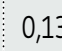

$\begin{array}{c:c:c:c}0,1327 & -0,2674 & 0,0953 & 0,1118 \\ a & a & a & a \\ 0,3872 & 0,0074 & 0,3646 & 0,3481\end{array}$

el nutriente

Cantidad riesgosa para la salud

$\begin{array}{l:l:l}24 & 24 & 35\end{array}$

32

21

12

13

Más de lo necesario/sobre el

17

nivel permitido

Tiene añadido el nutriente (1)
11

7

0

6

34

14

0

28

$\begin{array}{llllll}-0,0856 & 0,0148 & 0,1056 & -0,0869\end{array}$

No se/No entiendo

14

$$
3
$$

5

17

6

2

0

$\operatorname{Otros}^{(4)}$

23

0

100

100

100

100

N: Total por categoría; \%: porcentaje; IC: Intervalo de Confianza.

(1) Corresponde al número de consumidores.

(2) \%: Se une el " $\mathrm{N}$ " $\mathrm{y}$ " $\%$ ", puesto que el total son 100 consumidores.

(3) IC: $95 \%$.

(4) Otros: "grasas que aumentan colesterol", "grasas procesadas", "frituras", "grasa de origen animal", "grasas no digeribles", "aceites", "comida chatarra", "de origen animal".

no interpretativo (numérico, que se basa en la ingesta de referencia (RI)) mostrando una mayor eficacia en la elección de los alimento más saludables ${ }^{35,38}$. Otro estudio en Ecuador, señala que un $30,7 \%$ de los encuestados lee las etiquetas nutricionales y el $26,2 \%$ considera las indicaciones que ellas entregan ${ }^{39}$.

En Alemania se ha empezado a usar el etiquetado frontal Nutri-Score, con impacto en el mercado local y Europa, en la comercialización desde y hacia Alemania ${ }^{38}$. En otros estudios se han realizado evaluaciones en el impacto en la compra de productos más saludables, uno de ellos realizado en padres con hijos/as de 3 y 12 años que frente a tres tipos de etiquetados (semáforo, con el contenido energético y sin etiquetar) no logró evidenciar la efectividad del etiquetado $0^{40,41}$; otro estudio que comparó tres etiquetados (semáforo, de advertencia y sin etiquetado) observó que el de advertencia impactó sólo en la selección de los productos altos en sodio, similar al de bebidas azucaradas que comparó el de advertencias con el de resumen, observando que el de advertencia es más eficiente en disminuir la elección de las bebidas azucaradas ${ }^{41,42}$; Nutri-Score de Francia y Health Star Rating de Australia mostró que el Nutri-Score permitía una mejor percepción de los productos saludables 35,38 . 
Ley 20.606: Efectos en el conocimiento de etiquetado nutricional en consumidores de un supermercado en Valparaíso de Chile: estudio descriptivo, cuanticualitativo, antes y después de 5 meses de la implementación de la ley

Figura 4. Cambios en la selección de los alimentos de los consumidores según la cantidad de rótulos que presentan, posterior a la entrada en vigencia de la ley 20.606. Diciembre 2016. Valparaíso. Chile.

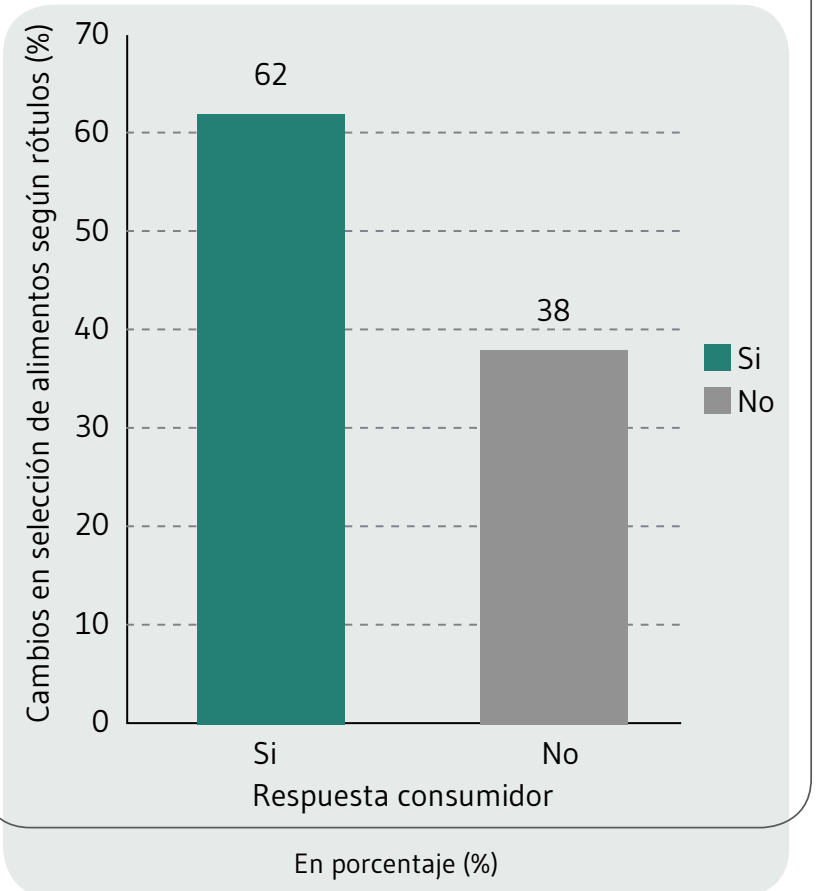

Un estudio de Chile realizado entre los años 2015 y 2017, mostró una disminución del $23,7 \%$ en la compra de bebidas con alto contenido de azúcares (22,8mL per cápita/día) luego de la implementación de la ley 20.60642. Finalmente, en el caso de nuestro estudio sus resultados son coincidentes respecto a que el etiquetado de advertencia contribuye a una mejor comprensión e identificación de la calidad nutricional de los productos y en este aspecto, lo que se observa en la categoría "no sé/no entiendo" disminuye para todos los rótulos "ALTOS EN" en la encuesta 2 y en la interpretación que el alimento tiene "más de lo necesario/sobre el nivel permitido" sólo para los rótulos " ALTO EN" "AZÚCAR" y "ALTO EN" "SODIO" podría explicar también lo observado en el estudio sobre la disminución de los consumidores en la elección de las bebidas azucaradas en Chile $^{42}$.

En este estudio, el 95\% promedio de ambas encuestas, señala que falta educación sobre el nuevo etiquetado y sugieren modificar el logo o tamaño. Un estudio realizado en 2017 por Nutricionistas y estudiantes de Nutrición y Dietética de Chile, indica que entre un $99 \%$ y un $99,2 \%$ respectivamente, presentan un buen conocimiento y una percepción favorable de la ley, haciendo énfasis en que se debe mejorar la aplicación de la ley en los establecimientos educacionales ${ }^{43}$.
En el presente estudio, la muestra por conveniencia y su tamaño es una de las limitantes que no permiten extrapolar los resultados a la población de la comuna de Valparaíso. En ese sentido sería un aporte al conocimiento del impacto de los rótulos "ALTOS EN" con una muestra representativa.

Como limitantes de este estudio se pueden señalar el tamaño de la muestra estudiada (200 personas) por el horario, tiempo para aplicarla o que limita sus resultados a los consumidores del supermercado y no pueden extrapolarse a la población de Valparaíso. Una fortaleza de este estudio es disponer de datos cualitativos sobre el conocimiento e interpretación de los rótulos "ALTOS EN" en la primera etapa de la ley que puede orientar otros estudios en el área.

\section{CONCLUSIONES}

La lectura del etiquetado frontal de alimentos a los cinco meses de la implementación de la primera etapa de la ley de etiquetado aumenta levemente. El etiquetado frontal de los alimentos y los sellos octagonales permiten una mejor visualización de la composición nutricional de los alimentos pero los consumidores participantes de este estudio a cinco meses de la implementación de la primera etapa de la ley no logran la interpretación de todos los rótulos "ALTOS EN". Los rótulos "ALTO EN" "AZÚCAR" y "SODIO" son los que logran ser más interpretados por los consumidores. Respecto al daño en la salud del consumo de productos "ALTOS EN", los consumidores logran un bajo reconocimiento de la relación entre su consumo y enfermedades. Esta estrategia requiere incorporar la educación alimentaria y nutricional sistemática en los consumidores para que el etiquetado frontal de advertencia y sus rótulos sean comprendidos y mejor interpretados por la población. Se requieren más estudios cualitativos sobre la comprensión objetiva, percepción e interpretación de la información del etiquetado frontal de los alimentos por los consumidores.

\section{AGRADECIMIENTOS}

A Lissette Pérez Bocaz, Nutricionista, ayudante académica en formación del Departamento de Nutrición de la Facultad de Ciencias de la Salud de la Universidad de Playa Ancha, por la lectura del artículo y sugerencias. 
Ley 20.606: Efectos en el conocimiento de etiquetado nutricional en consumidores de un supermercado en Valparaíso de Chile: estudio descriptivo, cuanticualitativo, antes y después de 5 meses de la implementación de la ley

\section{CONTRIBUCIÓN DE AUTORÍA}

MCM: diseño estudio, elaboración instrumentos, análisis de datos, preparación artículo. MA: elaboración instrumentos, aplicación instrumentos, base de datos. YR: elaboración instrumentos, aplicación instrumentos, base de datos.

\section{FINANCIACIÓN}

Este estudio no dispuso de recursos económicos para su realización.

\section{$\longrightarrow$ \\ CONFLICTO DE INTERESES}

Las autoras expresan que no existen conflictos de interés al redactar el manuscrito.

\section{REFERENCIAS}

(1) OECD. (2017). Obesity Update. 2019, de Organización para la Cooperación y el Desarrollo Económico (OCDE) Sitio web: http://www.oecd.org/health/obesity-update.htm

(2) Departamento de Epidemiología. Encuesta Nacional de Salud: primeros resultados. [Internet]. División de Planificación Sanitaria, Subsecretaría de Salud Pública, MINSAL; 2017. Disponible en: https://www.minsal.cl/wp-content/ uploads/2017/11/ENS-2016-17_PRIMEROS-RESULTADOS.pdf

(3) Ministerio de Salud, Departamento de Epidemiología. Encuesta Nacional de Salud: primeros resultados. Tomo I. [Internet]. Chile: División de Planificación Sanitaria, Subsecretaría de Salud Pública, MINSAL; 2010. 1064 p. Disponible en: https:// www.minsal.cl/wp-content/uploads/2017/11/ENS-2016-17_ PRIMEROS-RESULTADOS.pdf

(4) Unidad de Estudios JUNAEB. Informe Mapa Nutricional 2017. Situación nutricional de los preescolares y escolares de establecimientos municipalizados y particulares subvencionados del país. [Internet]. Chile: JUNAEB; 2018 p. 17. Report No.: R-SGC-IE001. Disponible en: https://www.junaeb. cl/wp-content/uploads/2013/03/Informe-Mapa-Nutricional2017-FINAL.pdf

(5) Lira M. Informe Mapa Nutricional 2018: Situación nutricional de los párvulos y escolares de establecimientos escolares con financiamiento público del país. [Internet]. Chile: Unidad de Estudios JUNAEB; 2019 p. 21. Disponible en: https://www.
junaeb.cl/wp-content/uploads/2019/12/Informe-MapaNutricional-2018.pdf

(6) World Health Organization. Non communicable diseases [Internet]. World Health Organization. 2018. Disponible en: https://www.who.int/news-room/fact-sheets/detail/ noncommunicable-diseases

(7) Consulta Mixta OMS/FAO de Expertos en Régimen Alimentario, Nutrición y Prevención de, Enfermedades Crónicas. Dieta, nutrición y prevención de enfermedades crónicas. [Internet]. Ginebra: OMS; 2003 p. 152. (Serie de Informes Técnicos OMS). Report No.: 916. Disponible en: https://apps.who.int/ iris/bitstream/handle/10665/42755/WHO_TRS 916 spa. pdf? sequence $=1$

(8) Gakidou E, Afshin A, Abajobir AA, Abate KH, Abbafati C, Abbas $\mathrm{KM}$, et al. Global, regional, and national comparative risk assessment of 84 behavioural, environmental and occupational, and metabolic risks or clusters of risks, 19902016: a systematic analysis for the Global Burden of Disease Study 2016. Lancet. 16 de septiembre de 2017; 390(10100): 1345-422.

(9) Organización Panamericana de la Salud. Plan de acción para la prevención y el control de las enfermedades no transmisibles en las Américas 2013-2019 [Internet]. Washington DC: OPS/ OMS; 2014. 64 p. Disponible en: https://www.paho.org/hq/ dmdocuments/2015/plan-accion-prevencion-control-entamericas.pdf

(10) Organización Panamericana de la Salud. Modelo de perfil de nutrientes de la Organización Panamericana de la Salud [Internet]. Washington DC: OPS/OMS; 2016. 38 p. Disponible en: https://iris.paho.org/bitstream/ handle/10665.2/18622/9789275318737 spa.pdf

(11) Organización Panamericana de la Salud. Alimentos y bebidas ultraprocesados en América Latina: tendencias, efecto sobre la obesidad e implicaciones para las políticas públicas. [Internet]. Washington DC: OPS/OMS; 2015. 76 p. Disponible en: https://iris.paho.org/bitstream/ handle/10665.2/7698/9789275318645_esp.pdf

(12) Cediel G, Reyes M, Louzada ML da C, Steeele EM, Monteiro CA, Corvalán $C$, et al. Ultra-processed foods and added sugars in the Chilean diet (2010). Public Health Nutrition. Enero de 2018; 21(1): 125-33.

(13) Crovetto M, Uauy R, Martins AP, Moubarac JC, Monteiro C. Household availability of ready-to-consume food and drink products in Chile: impact on nutritional quality of the diet. Rev Med Chil. Julio de 2014; 142(7): 850-8.

(14) Crovetto M, Uauy R. Evolución del gasto en alimentos procesados en la población del Gran Santiago en los últimos 20 años. Rev Med Chil. Marzo de 2012; 140(3): 305-12.

(15) World Health Organization. Guiding principles and framework manual for front-of-package labelling for promoting healthy diet [Internet]. WHO; 2019. Disponible en: https://www. who.int/nutrition/publications/policies/guiding principleslabelling-promoting-healthydiet.pdf?ua=1

(16) Ministerio de Salud, Subsecretaría de Salud Pública. Sobre composición nutricional de los alimentos y su publicidad [Internet]. 2012. Disponible en: https://www.minsal.cl/sites/ default/files/LEY-20606_06-JUL-2012.pdf

(17) República de Chile, División Jurídica. Reglamento Sanitario de los Alimentos. Dto. N 977/96 (D.OF. 13.05.97) [Internet]. 
Ley 20.606: Efectos en el conocimiento de etiquetado nutricional en consumidores de un supermercado en Valparaíso de Chile: estudio descriptivo, cuanticualitativo, antes y después de 5 meses de la implementación de la ley

Ministerio de Salud de Chile; 2015. Disponible en: https://www. minsal.cl/wp-content/uploads/2017/04/DECRETO_977_96_ actualizado_a-octubre-2016.pdf

(18) Ministerio de Salud, Subsecretaría de Salud Pública. Modifica Decreto Supremo N977, de 1996, Reglamento Sanitario de los Alimentos [Internet]. 2015. Disponible en: https:// www.minsal.cl/sites/default/files/decreto_etiquetado_ alimentos_2015.pdf

(19) Rodríguez Osiac L, Pizarro Quevedo T, Rodríguez Osiac L, Pizarro Quevedo T. Ley de Etiquetado y Publicidad de Alimentos: Chile innovando en nutrición pública una vez más. Revista chilena de pediatría. Octubre de 2018; 89(5): 579-81.

(20) Reyes M, Garmendia ML, Olivares S, Aqueveque C, Zacarías I, Corvalán C. Development of the Chilean front-of-package food warning label. BMC Public Health. 8 de julio de 2019; 19(1): 906.

(21) Corvalán C, Reyes M, Garmendia ML, Uauy R. Structural responses to the obesity and non-communicable diseases epidemic: Update on the Chilean law of food labelling and advertising. Obesity Reviews. 2019; 20(3): 367-74.

(22) La Brújula Salud. Encuesta sobre etiquetado de alimentos 2016. [Internet]. Universidad San Sebastian; 2016. Disponible en: https://www.uss.cl/newsletter-uss/wp-content/uploads/ sites/19/2016/07/Encuesta-Ley-de-Etiquetado-de-Alimentos. pdf

(23) Temas de interés. [Internet]. Universidad San Sebastian. Instituto de Políticas Públicas en Salud de la Universidad San Sebastián (IPSUSS); 2016. Disponible en: https://www. uss.cl/medicina/50-quienes-leen-etiquetado-los-alimentosreconoce-influye-compra/

(24) Universidad de Chile. Informe de resultados. Descripción de las percepciones y actitudes de los/as consumidores respecto a las medidas estatales en el marco de la implementación del Decreto 13/15. [Internet]. Chile: Instituto de la Comunicación e Imagen (ICEI); 2017. Disponible en: https://www.minsal.cl/ wp-content/uploads/2017/01/Informe-Percepci\%C3\%B3nConsumidores-ICEI.pdf

(25) Subsecretaría de Salud Pública. Informe de Evaluación de la implementación de la Ley sobre composición nutricional de los alimentos y su publicidad [Internet]. Chile: División de Políticas Públicas Saludables y Promoción. Departamento de Nutrición y Alimentos; 2018 p. 20. Disponible en: https:// www.minsal.cl/wp-content/uploads/2018/05/InformeImplementaci\%C3\%B3n-Ley-20606-febrero-18-1.pdf

(26) Olivares-Cortés S, Araneda-Flores J, Morales-IIlanes G, Leyton-Dinamarca B, Bustos-Zapata N, Hernández-Moreno $M-A$, et al. Actitudes de escolares chilenos de distinto nivel socioeconómico al inicio de la implementación de la ley que regula la venta y publicidad de alimentos altos en nutrientes críticos. Nutr Hosp. Abril de 2017; 34(2): 431-8.

(27) Kanter R, Reyes M, Swinburn B, Vandevijvere S, Corvalán C. The Food Supply Prior to the Implementation of the Chilean Law of Food Labeling and Advertising. Nutrients. Enero de 2019; 11(1): 52.

(28) Correa T, Fierro C, Reyes M, Dillman Carpentier FR, Taillie LS, Corvalan C. Responses to the Chilean law of food labeling and advertising: exploring knowledge, perceptions and behaviors of mothers of young children. Int ] Behav Nutr Phys Act. 13 de febrero de 2019; 16(1): 21.

(29) Santos-Antonio G, Bravo-Rebatta F, Velarde-Delgado P,
Aramburu A. Efectos del etiquetado nutricional frontal de alimentos y bebidas: sinopsis de revisiones sistemáticas. Rev Panam Salud Publica [Internet]. 12 de agosto de 2019; 43. Disponible en: https://www.ncbi.nlm.nih.gov/pmc/articles/ PMC6692502/.

(30) Risso Patrón V, Hansen E, Valle Ruidiaz A. Etiquetado nutricional frontal de alimentos [Internet]. Argentina: Secretaría de Gobierno de Salud, Ministerio de Salud y Desarrollo Social Presidencia de la Nación; 2018. 43 p. Disponible en: http://www.msal.gob.ar/images/stories/bes/ graficos/0000001380cnt-2019-06_etiquedato-nutricionalfrontal-alimentos.pdf

(31) Kaufer-Horwitz M, Tolentino-Mayo L, Jáuregui A, SánchezBazán K, Bourges H, Martínez S, et al. Sistema de etiquetado frontal de alimentos y bebidas para México: una estrategia para la toma de decisiones saludables. Salud Publica Mex. 28 de junio de 2018; 60(4): 479-86.

(32) Ministerio de Salud. Manual de advertencias publicitarias del Reglamento de la Ley No. 30021, Ley de promoción de la alimentación saludable para niños, niñas y adolescentes [Internet]. República del Perú.; 2017. Disponible en: ftp:// ftp2.minsa.gob.pe/normaslegales/2017/RM_N\%C2\%B0_6832017-MINSA.pdf

(33) Instituto Brasileiro de Defesa do Consumidor. Anvisa inicia oficialmente a revisão de normas de rotulagem nutricional [Internet]. idec. 2017. Disponible en: https://idec.org.br/ noticia/anvisa-inicia-oficialmente-revisao-de-normas-derotulagem-nutricional

(34) Roseman MG, Joung H-W, Littlejohn El. Attitude and Behavior Factors Associated with Front-of-Package Label Use with Label Users Making Accurate Product Nutrition Assessments. ] Acad Nutr Diet. 1 de mayo de 2018; 118(5): 904-12.

(35) Egnell M, Talati Z, Hercberg S, Pettigrew S, Julia C. Objective Understanding of Front-of-Package Nutrition Labels: An International Comparative Experimental Study across 12 Countries. Nutrients. Octubre 2018; 10: 1542. https://doi. org/10.3390/nu10101542

(36) Egnell M, Ducrot P, Touvier M, Allès B, Hercberg S, Kesse-Guyot $E$, et al. Objective understanding of Nutri-Score Front-OfPackage nutrition label according to individual characteristics of subjects: Comparisons with other format labels. PLOS ONE. 23 de agosto de 2018; 13(8): e0202095.

(37) Scarborough P, Matthews A, Eyles H, Kaur A, Hodgkins C, Raats $M M$, et al. Reds are more important than greens: how UK supermarket shoppers use the different information on a traffic light nutrition label in a choice experiment. Int J Behav Nutr Phys Act. 12 de diciembre de 2015; 12(1): 151.

(38) Szabo de Edelenyi F, Egnell M, Galan P, et al. Ability of the Nutri-Score front-of-pack nutrition label to discriminate the nutritional quality of foods in the German food market and consistency with nutritional recommendations. Arch Public Health. 2019; 77: 28. Disponible en: https://doi.org/10.1186/ s13690-019-0357-x.

(39) Ramos Padilla PD, Carpio Arias TV, Delgado López VC, Villavicencio Barriga VD, Andrade CE, Fernández-Sáez J. Actitudes y prácticas de la población en relación al etiquetado de tipo "semáforo nutricional" en Ecuador. Rev Esp Nutr Hum Diet. 2017; 21(2): 121-9. Disponible en: http://dx.doi. org/10.14306/renhyd.21.2.306 
Ley 20.606: Efectos en el conocimiento de etiquetado nutricional en consumidores de un supermercado en Valparaíso de Chile: estudio descriptivo, cuanticualitativo, antes y después de 5 meses de la implementación de la ley

(40) Dodds P, Wolfenden L, Chapman K, Wellard L, Hughes C, Wiggers $]$. The effect of energy and traffic light labelling on parent and child fast food selection: a randomised controlled trial. Appetite. 1 de febrero de 2014; 73: 23-30.

(41) Graham DJ, Lucas-Thompson RG, Mueller MP, Jaeb M, Harnack L. Impact of explained v. unexplained front-of-package nutrition labels on parent and child food choices: a randomized trial. Public Health Nutr. 2017; 20(5): 774-85.

(42) Taillie LS, Reyes M, Colchero MA, Popkin B, Corvalán C. An evaluation of Chile's Law of Food Labeling and Advertising on sugar-sweetened beverage purchases from 2015 to 2017: A before-and-after study. PLOS Medicine. 11 de febrero de 2020; 17(2): e1003015.

(43) Duran-Agüero S, Parra S, Ahumada D, Castro P, Brignardello J, Riedemann K, et al. Ley de alimentos: una mirada de los nutricionistas y estudiantes de nutrición y dietética de Chile. Rev Esp Nutr Hum Diet. 31 de diciembre de 2017; 21(4): 327-34. 